\title{
Gonadotropina coriónica en flujo vaginal para el diagnóstico de rotura prematura de membranas
}

\author{
Angélica Urdaneta-García ${ }^{1}$, Eduardo Reyna-Villasmil ${ }^{1}$, Jorly Mejia-Montilla ${ }^{1}$, Duly \\ Torres-Cepeda ${ }^{1}$, Joel Santos-Bolívar ${ }^{1}$, Nadia Reyna-Villasmil ${ }^{1}$, Ismael Suárez-Torres ${ }^{1}$, \\ Anny Cuevas-González. ${ }^{1}$
}

${ }^{1}$ Servicio de Obstetricia y Ginecología - Maternidad "Dr. Nerio Belloso", Hospital Central "Dr. Urquinaona”. Maracaibo, Estado Zulia. Venezuela.

\section{RESUMEN}

Objetivo: Establecer la eficacia diagnóstica de la determinación de gonadotropina coriónica en flujo vaginal para el diagnóstico de la rotura prematura de membranas. Método: Investigación de tipo prospectiva en muestra de 270 embarazadas que asistieron al Hospital Central "Dr. Urquinaona", Maracaibo, Venezuela. Los grupos consistieron en pacientes con rotura prematura de membranas (grupo $A ; n=135$ ) y embarazadas con membranas integras (grupo $B ; n=135$ ), consideradas como controles. Se evaluaron las características generales, valores de gonadotropina coriónica en flujo vaginal y efectividad diagnóstica. Resultados: La edad gestacional al momento de la determinación de las concentraciones de gonadotropina coriónica en flujo vaginal fue de $32,9 \pm 1,6$ semanas para el grupo A y 33,1 $\pm 1,9$ semanas para el grupo B $(p=n s)$. No se encontraron diferencias estadísticamente significativas en la edad materna y la frecuencia de paridad entre ambos grupos de tratamiento $(p=n s)$. Las pacientes del grupo A presentaron concentraciones significativamente más altas de gonadotropina coriónica en flujo vaginal $(697,4 \pm 382,4 \mathrm{mUl} / \mathrm{mL})$ comparado con las embarazadas del grupo B $(91,4 \pm 47,1 \mathrm{mUI} / \mathrm{mL} ; \mathrm{p}<0,0001)$. Un valor de corte de 100 $\mathrm{mUl} / \mathrm{mL}$ presentó un valor por debajo de la curva de 0,96 con una sensibilidad del 97,0\%, especificidad del $51,1 \%$, valor predictivo positivo del $66,5 \%$ y valor predictivo negativo del $94,5 \%$. Conclusión: La determinación de las concentraciones de gonadotropina coriónica en el flujo vaginal es una técnica diagnóstica útil para la rotura prematura de membranas.

\section{PALABRAS CLAVE: Gonadotropina coriónica, rotura prematura de membranas, líquido amniótico}

\section{SUMMARY}

Objective: To establish the diagnostical efficacy of chorionic gonadotropin in vaginal fluid determination for diagnosis of premature rupture of membranes. Method: A prospective research type was done with a sample of 270 pregnant women who assisted to obstetrics emergency at Hospital Central "Dr. Urquinaona", Maracaibo, Venezuela. Groups consisted in patients with premature rupture of membranes (group A; $n=135$ ) and pregnant women with intact membranes (group $B ; n=135$ ), considered as controls. General characteristics, values of chorionic gonadotropin in vaginal fluid and diagnostic efficacy were evaluated. Results: Gestational age at the moment of determination chorionic gonadotropin in vaginal fluid was $32.9 \pm$ 1.6 weeks in group $A$ and $33.1 \pm 1.9$ weeks in group $B(p=n s)$. There were no significant differences in maternal age and frequency of parity between groups $(p=n s)$. Patients in group $A$ showed significant higher concentrations of chorionic gonadotropin in vaginal fluid $(697.4 \pm 382.4 \mathrm{mUI} / \mathrm{mL})$ compared with pregnant women in group B $(91.4 \pm 47.1 \mathrm{mUI} / \mathrm{mL} ; \mathrm{p}<0.0001)$. A cut-off point of $100 \mathrm{mUl} / \mathrm{mL}$ showed a under the curve value of 0.96 with a sensitivity of $97.0 \%$, specificity of $51.1 \%$, positive predictive value of $66.5 \%$ and 
negative predictive value of $94.5 \%$. Conclusions: Determination of chorionic gonadotropin concentrations in vaginal fluid is a useful diagnosis tool for premature rupture of membranes.

\section{KEY WORDS: Chorionic gonadotropin, premature rupture of membranes, amniotic fluid}

\section{INTRODUCCIÓN}

La rotura prematura de membranas (RPM) se presenta en $5-15 \%$ de todos los partos a término y en $20-40 \%$ de los partos pretérminos. La complicación más común es la infección. Aunque los embarazos a término con RPM tienen riesgo de infección, la principal amenaza ocurre en los embarazos pretérminos. El riesgo de infección se incrementa en forma concomitante con un periodo de latencia prolongado (1).

La RPM es fácil de diagnosticar cuando se demuestra la salida de líquido amniótico a través del cuello pero es más difícil cuando existe la duda sobre si esta ha ocurrido. El fallo al identificar a estas pacientes puede llevar al fallo de la implementación de conductas obstétricas, mientras que el falso diagnóstico puede llevar a intervenciones inapropiadas como hospitalización e interrupción del embarazo.

El diagnóstico generalmente depende de una combinación de factores, incluyendo antecedentes de la paciente, examen físico, identificación del índice de líquido amniótico y la prueba de nitrazina $(2,3)$. Sin embargo, en los casos donde no existe RPM, los métodos tradicionales se han asociado con tasas de falsos positivos y falsos negativos de $17,4 \%$ y $9,4 \%$, respectivamente (4). Los resultados falsos positivos de la prueba de nitrazina se han asociado con cervicitis, vaginitis, contaminación vaginal por semen, orina alcalina y sangre.

La ausencia de una prueba no invasiva "ideal" para el diagnóstico de RPM ha llevado a la búsqueda de marcadores bioquímicos alternativos. Se han evaluado las concentraciones vaginales de diaminoaxidasa, prolactina, alfafetoproteína (AFP), fibronectina fetal y proteína 1 fijadora del factor de crecimiento similar a la insulina (IGFBP-1) (5-11). Sin embargo, la prolactina y la AFP no son marcadores útiles en la RPM debido a que sus concentraciones se sobreponen entre las embarazadas con y sin RPM (6-9). Más aún, la liberación crónica de fibronectina previo al parto en embarazadas con membranas intactas puede llevar a resultados falso positivos $(9,10)$. La IGFBP-1 tiene una sensibilidad de $74,4 \%$ y un valor predictivo negativo de $55,6 \%$ (11).

La gonadotropina coriónica (HCG) es una glicoproteína producida exclusivamente por el sinciciotrofoblasto en la placenta (12). Con la progresión del embarazo, las concentraciones promedio se incrementa en la circulación materna hasta aproximadamente $54.000 \mathrm{mUl} / \mathrm{mL}$ a las $8-12$ semanas de gestación. Posteriormente declina alcanzando una meseta aproximadamente a las 20 semanas. Estas concentraciones se mantienen alrededor de 12.000 $\mathrm{mUl} / \mathrm{mL}$ durante el tercer trimestre. Está presente en el líquido amniótico al igual que en la sangre y la orina materna en concentraciones que varían de 2.000 a $70.000 \mathrm{mUI} / \mathrm{mL}$ (12). También es secretada por las células vaginales y está presente en pequeñas concentraciones en el fluido vaginal.

La determinación de HCG en flujo vaginal es una prueba fácil, económica, rápida y no invasiva, que se puede realizar en pacientes hospitalizadas y ambulatorias. Estudios previos han reportado diferentes valores diagnósticos (13-15). El objetivo de la investigación fue establecer la eficacia diagnóstica de la determinación de las concentraciones de gonadotropina coriónica humana en flujo vaginal para el diagnóstico de la rotura prematura de membrana.

\section{PACIENTES Y MÉTODOS}

Investigación de tipo prospectivo realizado en el Hospital Central "Dr. Urquinaona" entre enero del 2010 y diciembre del 2012. La muestra fue probabilística intencional de pacientes en las que se recolectó la secreción vaginal después de obtener la aprobación del Comité de Ética del hospital y el consentimiento por escrito de las mujeres con embarazos de 20 a 36 semanas. La edad gestacional fue establecida por la fecha de última regla y confirmada por la evaluación ecográfica antes de las 14 semanas. Todas las mujeres seleccionadas presentaban embarazos simples.

Se excluyó a las embarazadas con menos de 20 semanas de embarazo, restricción del crecimiento intrauterino del feto, polihidramnios, alteraciones de la frecuencia cardiaca fetal, anomalías fetales, muerte fetal intrauterina o embarazo postérmino, enfermedades crónicas (diabetes, hipertensión arterial crónica o gestacional), diagnóstico de parto pretérmino, presencia de infección intrauterina (por ejemplo, corioamnionitis) o materna activa, presencia de sangrado o infección vaginal, relaciones sexuales en las 48 horas previas a la realización de la evaluación, neoplasias, enfermedades inmunológicas u obesidad. Así mismo, fueron excluidas las embarazadas con 
sangrado y flujo vaginal patológico y aquellas que se negaron a participar en el estudio.

Las pacientes fueron divididas en 2 grupos: grupo A o de estudio, pacientes con RPM confirmada por acumulo de líquido amniótico, prueba de helecho y papel de nitrazina positiva y grupo B o controles, pacientes sin RPM que fueron seleccionadas por tener la misma edad gestacional, evaluadas en el Servicio de Obstetricia y que asistieron a la consulta prenatal, sin enfermedad ni complicaciones. Una vez seleccionadas las pacientes para el estudio, se llenó una ficha de recolección de datos que incluyó: identificación de la paciente, antecedentes personales y gineco-obstétricos, control prenatal, edad de gestación (por fecha de última regla o ecografía del primer trimestre) y concentraciones de HCG en flujo vaginal. Todos los procedimientos y las evaluaciones ecográficas fueron realizados por un único investigador para eliminar las diferencias interobservador.

Para la obtención de la muestra de flujo vaginal, después de confirmar la ausencia de sangre, esta fue limpiada con una gasa estéril y se colocó un espéculo estéril en vagina. Se procedió a irrigar el fondo de saco vaginal posterior con $3 \mathrm{ml}$ de solución fisiológica estéril usando una jeringa de $10 \mathrm{ml}$; inmediatamente, con la misma jeringa, se aspiró todo el líquido, el cual se depositó en un tubo de ensayo estéril tapado. Se centrifugó la muestra de inmediato a $1.750 \mathrm{G}$ por 5 min y fue almacenada a $-20^{\circ} \mathrm{C}$ hasta que se determinó las concentraciones de HCG en forma cuantitativa. Las concentraciones se determinaron por inmunoensayo por electroquimioluminiscencia (Ortho-ClinicalDiagnosticsInc, EEUU.), con una sensibilidad analítica de $0,5 \mathrm{mUl} / \mathrm{mL}$ y un coeficiente de variación inter e intraensayo menor de $10 \%$. Se analizaron todas las muestras de flujo vaginal en un mismo laboratorio usando la misma técnica.

Los valores obtenidos se presentaron como promedio \pm desviación estándar. La prueba de la
U de Mann-Whitney fue utilizada para comparar las variables continuas. La precisión de las concentraciones de HCG para el diagnóstico de RPM se presenta en función de sensibilidad, especificidad, valor predictivo positivo y valor predictivo negativo. Se utilizó el análisis operador-receptor para determinar el mejor valor de corte. Se consideró $p<0,05$ como estadísticamente significativo.

\section{RESULTADOS}

Se seleccionó a un total de 270 embarazadas de las cuales el grupo A correspondió a las pacientes con RPM o casos $(n=135)$ y el grupo $B$ las embarazadas sin RPM que fueron seleccionadas como controles $(n=135)$. La edad gestacional al momento de la determinación de las concentraciones de HCG en flujo vaginal fue de $32,9 \pm 1,6$ semanas para el grupo $A$ y $33,1 \pm 1,9$ semanas para el grupo $B(p>0,05)$. No se encontraron diferencias estadísticamente significativas en la edad materna ni en la frecuencia de paridad entre ambos grupos de tratamiento $(p>0,05)$.

En la Figura 1 se muestran las concentraciones de HCG en flujo vaginal en las pacientes de ambos grupos. Las pacientes del grupo A presentaron concentraciones significativamente más altas de HCG $(697,4 \pm 382,4 \mathrm{mUl} / \mathrm{mL})$ comparadas con las embarazadas del grupo B $(91,4 \pm 47,1 \mathrm{mUl} / \mathrm{mL} ; \mathrm{p}$ $<0,0001$ ).

En la Figura 2 se muestra la curva operador receptor para la precisión diagnóstica de las concentraciones de HCG en el flujo vaginal para el diagnóstico de RPM. Un valor de corte de 100 $\mathrm{mUl} / \mathrm{mL}$ presentó un valor por debajo de la curva de 0,96 con una sensibilidad del 97,0\% (IC 95\%; $92,5-99,1 \%$ ), especificidad del $51,1 \%$ (IC $95 \%$; $42,3-59,8 \%$ ), valor predictivo positivo del $66,5 \%$ (IC $95 \% ; 59,4-73,0 \%$ ) y valor predictivo negativo del $94,5 \%$ (IC 95\%; 86,5-98,4\%).

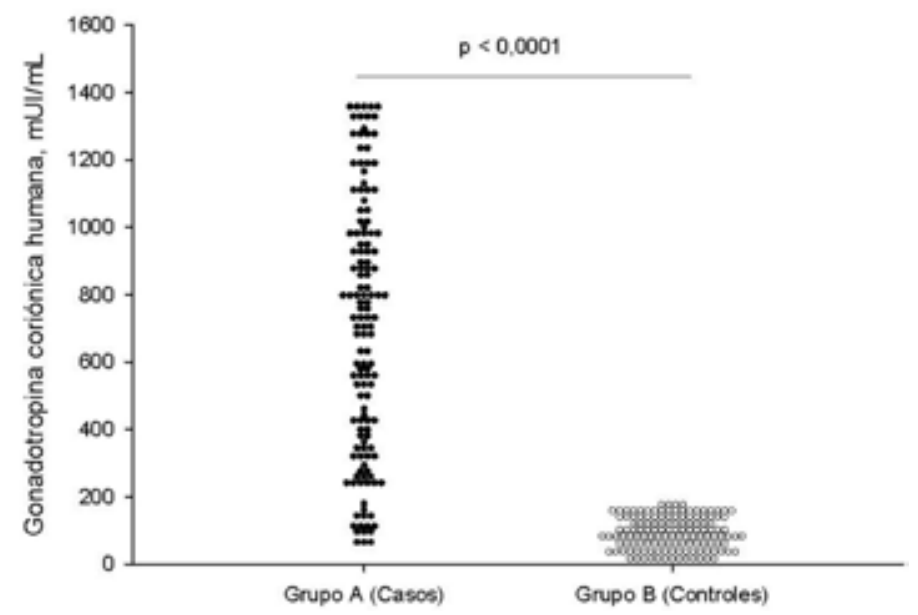

Figura 1. Concentración de gonadotropina coriónica en pacientes con y sin rotura prematura de membranas. 


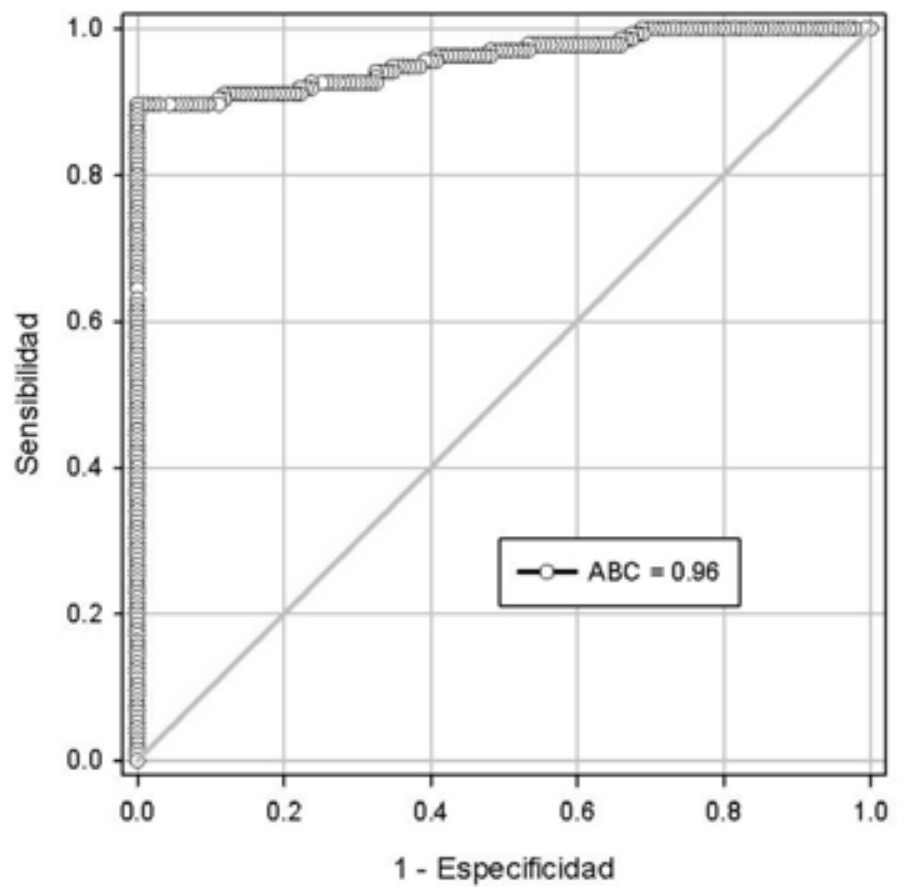

Figura 2. Curva operador receptor para las concentraciones de gonadotropina coriónica en flujo vaginal en el diagnóstico de rotura prematura de membranas.

\section{DISCUSIÓN}

La RPM está asociada con morbilidad infecciosa materno-fetal e inminente parto pretérmino o a término. Por estas razones el diagnóstico correcto es muy importante. Los antecedentes de la paciente, el interrogatorio y el examen físico generalmente son suficientes para el diagnóstico del $90 \%$ de los casos en los que se sospecha RPM. En la mayoría de los casos, el diagnóstico se realiza por el método tradicional: presencia de líquido amniótico en el fondo de saco, arborización del líquido amniótico y prueba de nitrazina (1)

Una técnica diagnóstica ideal debería ser aceptada tanto por las pacientes como por los médicos y debe ser apropiada, precisa y rápida. El uso de marcadores bioquímicos (AFP, diamino-oxidasa, prolactina o fibronectina fetal en el fluido vaginal) parecen ser métodos alternativos razonables para el diagnóstico de RPM $(8,11)$. Estos marcadores tienen ventajas y desventajas. Sin embargo, no han alcanzado la popularidad debido a la complejidad y costo para su determinación. Uno de los marcadores bioquímicos para el diagnóstico preciso es la $H C G$, ya que no requiere elementos diferentes a las pruebas habituales para el diagnóstico del embarazo, es de bajo costo y los resultados son rápidos.

La HCG está presente en el líquido amniótico, sangre materna y orina en valores de 2.000 a $70.000 \mathrm{mUl} / \mathrm{mL}$. También es secretada por las glándulas cervicales, por lo tanto debe estar presente en ciertas cantidades en el fluido vaginal (menos de $10 \mathrm{mUl} / \mathrm{mL}$ después de las 20 semanas) $(13,14)$. Después de la RPM, la gonadotropina coriónica puede ser detectada en altas concentraciones en los fluidos cervicovaginales (14).

Los resultados de la presente investigación demostraron que un valor de corte para HCG de $100 \mathrm{mUI} / \mathrm{mL}$ para el diagnóstico de RPM tiene sensibilidad de $97,0 \%$, especificidad de $51,1 \%$, valor predictivo positivo de $66,5 \%$ y valor predictivo negativo de $94,4 \%$. Anai y cols (14), demostraron una diferencia significativa en las concentraciones de HCG en el diagnóstico de RPM en el segundo y tercer trimestre, proponiendo un valor de corte de $50 \mathrm{mUl} / \mathrm{mL}$. Mangano y cols (16), propusieron un valor de corte de $100 \mathrm{mUI} / \mathrm{mL}$ en el fluido vaginal para la confirmación clínica de la RPM. Esim y cols (17), examinaron 141 pacientes y encontraron que 
el valor de corte óptimo era de $65 \mathrm{mUl} / \mathrm{mL}$ con un valor de sensibilidad, especificidad, valor predictivo positivo y negativo de $68 \%, 95 \%, 82 \%$ y $90 \%$, respectivamente. En otra investigación realizada por $\mathrm{Ni}$ y cols (18), la sensibilidad y especificidad de la HCG fue de $97,7 \%$ y $100 \%$, respectivamente. Kim y cols (13), estimaron un valor de corte de 39,8 $\mathrm{mUl} / \mathrm{mL}$ de la HCG en RPM en 120 pacientes; la sensibilidad, especificidad, valor predictivo positivo, valor predictivo negativo fue de $95,5 \%, 94,7 \%$, $91,3 \%$ y $97,3 \%$, respectivamente. Bahasadri y cols (19), encontraron un valor de corte de $79,5 \mathrm{mUl} / \mathrm{mL}$ con una sensibilidad de $85 \%$ y una especificidad de $84 \%$. Temel y cols (20), encontraron un valor de corte de $100 \mathrm{mUI} / \mathrm{mL}$ y los valores de sensibilidad, especificidad, valor predictivo positivo y negativo fueron de $71,2 \%, 100 \%, 100 \%$ y $61,5 \%$, respectivamente.

En Latinoamérica también se han hecho esfuerzos por establecer la utilidad de la HCG en el diagnóstico de RPM. Bufalino-Fianchino y cols (21), evaluaron 120 pacientes y demostraron que concentraciones en fluidos vaginales superiores a 17,1 $\mathrm{mUl} / \mathrm{mL}$ se relacionaron con RPM y presentaban valores de sensibilidad de $98,3 \%$, especificidad de $93,3 \%$, valor de predicción positivo $93,6 \%$ y valor de predicción negativo 98,5\%. Méndez-González y cols (22), refirieron un valor para HCG cualitativa por debajo del área de la curva de 0,939, con sensibilidad de $87 \%$, especificidad de $100 \%$, valor predictivo positivo de $100 \%$, valor predictivo negativo de $65 \%$, sin falsos positivos y falsos negativos de $12,3 \%$. Ramírez-Martínez y cols (23), evaluaron 175 pacientes y encontraron que los valores de sensibilidad, especificidad, valor predictivo positivo y valor predictivo negativo de $93,4 \%, 73,8 \%, 79,4 \%$ y $91,1 \%$, respectivamente.

Como se puede observar, se han reportado diferentes valores de corte, sensibilidad, especificidad y valores predictivos positivos y negativos para la HCG en fluido vaginal para el diagnóstico preciso de RPM. La presencia de estas diferencias se debe probablemente a diferentes razones: diferencias en el número de pacientes seleccionadas o la selección de pacientes con sangrado genital incluidas en algunos reportes. Como es conocido, los valores de corte varían en forma significativa en relación con el número de la muestra. También, los valores de HCG se modifican dependiendo del método de determinación al igual que en el momento que se produce la RPM (segundo o tercer trimestre) (13).

La determinación de HCG en flujo vaginal ofrece varias ventajas. La prueba puede ser realizada con la paciente en cama sin ningún tipo de instrumento especial y con pruebas de determinación co- mercial, suministrando una tasa de detección confiable y un costo razonable. El uso de esta prueba clínica puede reducir la frecuencia de tratamientos innecesarios cuando existen resultados conflictivos basados en otras pruebas diagnósticas. Pero se debe tener cuidado ya que la presencia de pequeñas cantidades de sangre en la muestra de fluido vaginal (debido a las altas concentraciones de gonadotropina coriónica en sangre) puede alterar los resultados de la prueba (17). También se debe considerar la presencia de infecciones y semen (24).

\section{CONCLUSIÓN}

La determinación de las concentraciones de gonadotropina coriónica humana en el flujo vaginal es una técnica diagnóstica útil y de bajo costo para el diagnóstico de la rotura prematura de membranas.

\section{REFERENCIAS}

1. Naylor CS, Gregory K, Hobel C. Premature rupture of the membranes: an evidence-based approach to clinical care. Am J Perinatol 2001;18:397-413.

2. Mercer BM. Preterm premature rupture of the membranes: current approaches to evaluation and management. Obstet Gynecol Clin North Am 2005;32:41128.

3. Dickinson F, Soltani H. Nitrazine yellow and pre-labour rupture of membranes. Pract Midwife 2008;11:48-52.

4. Bornstein J, Ohel G, Sorokin Y, ReapeKZ, Shnaider $\mathrm{O}$, Kessary-Shoham $\mathrm{H}$, et al. Effectiveness of a novel home-based testing device for the detection of rupture of membranes. Am J Perinatol 2009;26:45-50.

5. Broe D, Van Dongen J, Cowley D, Vacca A, Voreteliac $\mathrm{V}$, Maquire $\mathrm{D}$, et al. Detection of premature rupture of membranes by measuring diamine oxidase in vaginal fluid: false-negative results caused by obstetric antiseptic creams. Clin Chem 1992;38:784.

6. Phocas I, Sarandakou A, Kontoravdis A, Chryssicopoulos A, Zourlas PA. Vaginal fluid prolactin: a reliable marker for the diagnosis of prematurely ruptured membranes. Comparison with vaginal fluid alpha-fetoprotein and placental lactogen.Eur J ObstetGynecol Reprod Biol 1989;31:133-41.

7. Shahin M, Raslan H. Comparative study of three amniotic fluid markers in premature rupture of membranes: prolactin, beta subunit of human chorionic gonadotropin, and alpha-fetoprotein. Gynecol Obstet Invest 2007;63:195-9.

8. Gaucherand P, Guibaud S, Rudigoz RC, Wong A. Diagnosis of premature rupture of the membranes by the identification of alpha-feto-protein in vaginal secretions. Acta Obstet Gynecol Scand 1994;73:456-9.

9. Nageotte MP, Casal D, Senyei AE. Fetal fibronectin in patients at increased risk for premature birth. Am J Obstet Gynecol 1994;170:20-5.

10. Abdelazim IA. Fetal fibronectin (Quick Check fFNtest $(囚)$ for detection of premature rupture of fetal 
membranes. Arch Gynecol Obstet 2013;287:205-10

11. Akercan F, Cirpan T, Kazandi M, Terek MC, Mgoyi L, Ozkinay $\mathrm{E}$. The value of the insulin-like growth factor binding protein-1 in the cervical-vaginal secretion detected by immunochromatographic dipstick test in the prediction of delivery in women with clinically unconfirmed preterm premature rupture of membranes. Eur $\mathrm{J}$ Obstet Gynecol Reprod Biol 2005;121:159-63.

12. Kletzky OA, Rossman F, Bertolli SI, Platt LD, Mishell DR. Dynamics of human chorionic gonadotropin, prolactin, and growth hormone in serum and amniotic fluid throughout normal human pregnancy. Am J Obstet Gynecol 1985;151:878-84.

13. Kim YH, Park YW, Kwon HS, Kwon JY, Kim BJ.Vaginal fluid beta-human chorionic gonadotropin level in the diagnosis of premature rupture of membranes. Acta Obstet Gynecol Scand 2005;84:802-5.

14. Anai T, Tanaka Y, Hirota Y, Miyakawa I. Vaginal fluid hCG levels for detecting premature rupture of membranes. Obstet Gynecol 1997;89:261-4.

15. Li HY, Chang TS.Vaginal fluid creatinine, human chorionic gonadotropin and alpha-fetoprotein levels for detecting premature rupture of membranes. Zhonghua Yi XueZaZhi (Taipei) 2000;63:686-90

16. Mangano B, Diani F, Faccini G, Zatti N, Zardini E. Proposal of a new test for the diagnosis of PROM based on the determination of hCG in the washing fluid of the posterior vaginal fornix. Minerva Ginecol 2000;52:185-8.

17. Esim E, Turan C, Unal O, Dansuk R, Cengizglu B. Diagnosis of premature rupture of membranes by identification of beta-HCG in vaginal washing fluid. Eur J Obstet Gynecol Reprod Biol 2003;107:37-40.
18. Ni CY, JiaWX, Yi WM, FengLH, Yu LZ. Practicability of using vaginal fluid markers in detecting premature rupture of membranes. Ann Clin Biochem 2003;40:542-5.

19. Bahasadri S, Kashanian M, Khalili S. Evaluation of vaginal fluid $\beta$-human chorionic gonadotrophin for the diagnosis of preterm premature rupture of membranes. J Obstet Gynaecol Res 2013;39:777-82.

20. Temel O, Çöğendez E, Selçuk S, ReşitAsoğlu N, Kaya E. $\beta$-human chorionic gonadotropin assay in vaginal washing fluid for the accurate diagnosis of premature rupture of membranes during late pregnancy. J Turkish-German Gynecol Assoc 2013;14:201-4.

21. Bufalino-Fianchino G, Aponte-Cubillán A, CarrilloGarcía H, Bello F, Fabrega-Trueba R, Adrián-Pineda C. B-hCG en fluidos vaginales como marcador de rotura prematura de membranas. Rev Obstet Ginecol Venez 2003;63:181-6

22. Méndez-González J, Aguirre-Ramos G, ÁlvarezValero R, Velázquez-Magaña M, Rojas-Poceros G. Hormona gonadotropina coriónica humana vaginal versus cristalografía y papel de nitrazina para el diagnóstico de ruptura prematura de membranas. AnMed (Mex) 2007;52:22-6.

23. Ramírez-Martínez JJ, Soria-López JA, Ambriz-López $R$, Iglesias-Benavides JL. Comparación entre dos pruebas diagnósticas de rotura prematura de membranas. Ginecol Obstet Mex 2012;80:195-200.

24. Cooper AL, Vermillion ST, Soper DE. Qualitative human chorionic gonadotropin testing of cervico vaginal washings for the detection of preterm premature rupture of membranes. Am J Obstet Gynecol 2004;191:593-6. 